CAHIERS DE

NARRATOLOGIE
Cahiers de Narratologie

Analyse et théorie narratives

$10.2 \mid 2001$

La voix narrative

\title{
Voix sans issue (l'exemple de Charrue, de Robert Pinget)
}

\section{Frank Wagner}

\section{(2) OpenEdition}

1 Journals

Édition électronique

URL : http://journals.openedition.org/narratologie/10171

DOI : 10.4000/narratologie. 10171

ISSN : 1765-307X

Éditeur

LIRCES

\section{Édition imprimée}

Date de publication : 1 janvier 2001

Pagination : 495-508

ISBN : 2914561032

ISSN : 0993-8516

\section{Référence électronique}

Frank Wagner, « Voix sans issue (l'exemple de Charrue, de Robert Pinget) », Cahiers de Narratologie [En ligne], 10.2 | 2001, mis en ligne le 01 janvier 2001, consulté le 11 juin 2020. URL : http://

journals.openedition.org/narratologie/10171; DOI : https://doi.org/10.4000/narratologie.10171 


\title{
VOIX SANS ISSUE (L'EXEMPLE DE CHARRUE, DE ROBERT PINGET)
}

\author{
Frank WAGNER
}

"Pour sortir d'une impasse il faut en prendre une autre. Monsieur Songe. »

Certains linguistes estiment que pour comprendre le sens d'un message il est nécessaire de connaître son origine locutive, a fortiori s'il relève du mode discursif, c'est-à-dire si les empreintes du procès d'énonciation abondent dans l'énoncé. Reprenant en grande partie cette hypothèse à son compte, Gérard Genette insistait, en 1972, sur la nécessité pour la poétique de s'intéresser, sur le modèle des travaux linguistiques de Jakobson et Benveniste, à «l'instance productrice du discours narratif, $[\ldots]$ la narration $» 1$. Onze ans plus tard, esquissant un bilan critique de son "Essai de méthode », il signalait que ce chapitre de la voix narrative avait provoqué les discussions pour lui les plus cruciales, à propos de la catégorie de la personne.

28 ans après Figures III, 17 ans après Nouveau Discours du récit ${ }^{2}$, ces questions semblent n'avoir rien perdu de leur vivacité ni de leur pertinence. Bien sûr, certains ont $\mathrm{pu}$, depuis lors, dénoncer dans les efforts pour comprendre qui parle dans le texte de fiction "la tarte à la crème [...] d'un certain questionnement contemporain $»^{3}$, mais peut-être

${ }^{1}$ Gérard GENETTE, Figures III, Paris, Seuil, 1972, coll. « Poétique », p. 226.

${ }^{2}$ Gérard GENETTE, Nouveau Discours du récit, Paris, Seuil, 1983, coll. «Poétique ».

3 Laurent ADER, «Pinget polyphone (Note sur l'écriture et l'altérité dans L'Ennemi et Mahu ou le Matériau) ", Paris, Poétique $\mathrm{n}^{\circ} 79$, septembre 1989 , p. 327. 
est-ce faute d'y avoir goûté avec une suffisante assiduité. Car cette problématique ne cesse de rebondir, tant du fait de théoriciens, comme Lucien Dällenbach qui, dès 1980, signalait " l'effraction de la clôture structuraliste » ${ }^{4}$ que d'écrivains, comme Alain Robbe-grillet, qui insistait en 1984 sur l'urgence " de s'interroger à nouveau sur le rôle ambigu que jouent, dans le récit moderne, la représentation du monde et l'expression d'une personne, qui est à la fois un corps, une projection intentionnelle et un inconscient. ${ }^{5}$

En effet, qu'il s'agisse ou non d'un dessein délibéré de la part de Genette, force est de constater que l'attention rigoureuse et exclusive portée à la scène énonciative, définie par principe comme hermétique, a joué un rôle non négligeable dans l'édification de cette clôture, en raison du bannissement de l'auteur et des lecteurs dans le biographique et le socio-historique, c'est-à-dire hors de la sphère de la poétique. On le sait, en préambule à son étude de la voix narrative, Genette pose avec autorité une différence stricte entre instance narrative et instance littéraire, résumée par une "formule-choc »: " Marcel n'est pas Proust» (op. cit., p. 236), et peut ainsi affirmer que, plus généralement, «la situation narrative d'un récit de fiction ne se ramène jamais à sa situation d'écriture » (ibid., p. 226). Il défend ainsi, après Roland Barthes et Michel Foucault, une conception rigoureusement immanentiste et anti-intentionnaliste de la littérature.

Toutes les dates qui ont précédé visaient à désigner implicitement cette assurance comme partiellement conjoncturelle, puisqu'elle reconduit un positionnement intensément polémique hérité d'une opposition dédoublée à la critique impressionniste comme à la philologie de Lanson et de ses successeurs. Etayé par cette analyse, mon propos ne consistera pas pour autant à affirmer que Marcel est Proust, tout

4 Lucien DÄLlENBACH, «Réflexivité et lecture", Revue des Sciences Humaines, tome XLIX, janvier-mars 1980, p. 23 : "L'effraction de la clôture structuraliste a eu notamment pour effet de remettre à l'ordre du jour les problèmes de la réception et de la lecture, qu'un modèle du texte fondé sur la dichotomie saussurienne langue/parole ne permettait pas de prendre en compte."

5 Alain ROBBE-GRILLET, Le Miroir qui revient, Paris, Minuit, 1984, p. 12 . 
Proust, rien que Proust ou un peu Proust, mais à me demander si, dans le domaine de la fiction littéraire, il n'est pas possible de diagnostiquer, en prenant momentanément appui sur la catégorie poéticienne de la voix narrative, quelque chose comme une intention sans conscience. En d'autres termes, je me propose, au risque (assumé) de l'hétérodoxie sinon de l'hérésie proprement dite, de mettre la typologie de Genette et de ses épigones à l'épreuve d'une micro-lecture empirique, dans l'espoir d'ausculter ses capacités opératoires comme ses manques éventuels, qu'il faudra bien tenter le cas échéant de suppléer - sans pour autant perdre de vue les implications épistémologiques et idéologiques d'une telle tentative de « remédiation ».

\section{Charrue : «ni chou, ni rave ${ }^{6}$}

J'ai choisi, dans ce dessein, une œuvre légèrement postérieure à Nouveau Discours du récit: Charrue (1985) de Robert Pinget ${ }^{7}$. Il s'agit d'un texte de fiction, bref, fragmentaire, mais génériquement inclassable - l'assignation d'une identité générique unique et relevant d'une tradition antérieure y faisant l'objet d'une contestation soigneusement orchestrée par le recours à des séquences métatextuelles récurrentes et, comme souvent chez Pinget, contradictoires.

Mais pour les pingétiens, il s'agit tout de même d'un des trois carnets de monsieur Songe, personnage révélé en cette même année 1985 par la parution d'un ouvrage éponyme, mais déjà présent dans Le Harnais, publié en 1984. Le Harnais, Charrue, Du Nerf: ce système titulaire incite d'emblée à relativiser les certitudes immanentistes. Le Harnais constitue une partie du harnachement qui permet de tirer la Charrue, mais Monsieur Songe, écrivain («écrivassier»,

${ }^{6}$ Charrue, p. 31 : «D'une chose mal définie, [il dira] ça n’est ni chou ni rave."

${ }^{7}$ Robert PINGET, Charrue, Paris, Minuit, 1985.

Références bibliographiques des autres ouvrages de Robert Pinget analysés ou cités : Quelqu'un, Paris, Minuit, 1965 / Le Harnais, Paris, Minuit, 1984 / Monsieur Songe, Paris, Minuit, 1985 / L'Ennemi, Paris, Minuit, 1987 / Du Nerf, Paris, Minuit, 1990. 
« écrivailleur ${ }^{8}$ ) vieillissant et dégoûté doit s'exhorter à la tâche : Du Nerf ! Je prendrai donc principalement appui sur Charrue, volume tout de même autonomisé non seulement par les contingences éditoriales mais par sa structure circulaire et un dispositif métatextuel spécifique (les «notes roman »), mais m'autoriserai un recours ponctuel au réseau supratextuel et transcendant qui vient d'être défini - ce qui, déjà, revient à ne pas chercher le sens dans le seul espace délimité par les frontières du texte : du texte à l'auvre.

\section{Crise de voix}

Si tout lecteur de Charrue se trouve confronté, qu'il maîtrise ou non cette terminologie, au problème de la voix narrative, c'est que l'exploitation de ressources variées mais congruentes y contribue à un consciencieux brouillage de l'origine énonciative. Ainsi de la mise en place d'une intertextualité plurielle, hétérogène, composite, qui incite à considérer l'instance en charge de la parole narrative comme un narrateur ventriloqué, c'est-à-dire non pas seulement parlant mais parlé - par une multiplicité de voix, parfois discordantes ou dissonantes.

\section{Polyvocalité}

Faute de temps, je ne peux consacrer à ces phénomènes les minutieuses analyses qu'ils mériteraient, aussi me contenterai-je d'affirmer la prolifération dans Charrue des commérages, lieux communs, stéréotypes et expressions parémiologiques les plus variés ${ }^{9}$, voisinant avec une réappropriation

${ }^{8}$ Michèle PRAEGER, Les Romans de Robert Pinget (Une Ecriture des possibles), Lexington, Kentucky, French Forum Publishers, 1987, p. 26 et 30.

9 S'accommoder du désastre du langage constitue l'une des préoccupations de Pinget, dont les divers narrateurs doivent composer avec la prolifération parasite des expressions «toutes faites". Accumulation commentée et revendiquée à la faveur d'une séquence métatextuelle - « Il décide, n'ayant plus rien à perdre, d'utiliser sur ses vieux jours des expressions anciennes et régionales qui lui sont familières. " (Charrue, p. 30) - qu'on peut interpréter comme l'énoncé d'un principe esthétique. C'est la banalité du langage dans sa frange la plus désastreuse qui doit être assumée: le narrateur 
ironiquement distanciée du parlé des moralistes français du 17 ème siècle ${ }^{10}$, comme avec un intertexte gidien - plus précisément référencé aux Faux-monnayeurs ${ }^{11}$; sans oublier la convocation de l'ombre tutélaire de Paul Valéry ${ }^{12}$.

Ce hâtif survol permet de constater la multiplication des voix dans Charrue, voix qui parlent le narrateur plutôt qu'il ne les parle. Mais je n'en conclurai pas pour autant à la

démissionnaire choisit d'utiliser des mots vides parce que le langage est vide (l'insignifiance de ce qui est écrit dupliquant celle de l'acte d'écrire), parce que rien ne mérite d'être raconté, ou plutôt ne peut plus l'être.

10 Cette autre relation intertextuelle se nourrit bien sûr de similitudes formelles, puisque Charrue constitue un recueil, un assemblage d'aphorismes, maximes, confessions et notes diverses, et relève ainsi d'un genre fragmentaire, éclaté. En outre, l'inscription dans cette tradition est confirmée par les thèmes récurrents de la morale, de la vertu, des instincts bons ou mauvais ; par le recours aux ressources de la citation (le plus souvent implicite); par la réappropriation ironique du style des moralistes (archaïsmes syntaxiques et effets de symétrie); et de nouveau par la production de commentaires métatextuels. Ce nouvel intertexte entre dans un rapport d'opposition contrastive avec les clichés et expressions parémiologiques, mais les rejoint dans la dénonciation de l'impossible linéarité du récit, jugée arbitraire et fallacieuse.

11 Comme Gide, Pinget peut se poser comme Inquiéteur dans la mesure où il joue systématiquement du "paradoxe ou de la multiplicité du sens " (Du Nerf, p. 45) et vise à mettre en doute les certitudes des lecteurs, à maintenir indéfiniment en suspens les réponses aux questions posées : sens étymologique d'Eroneia. Mais c'est surtout la détection de similitudes nombreuses avec Les Faux-monnayeurs (Paris, Gallimard, 1949, réédité dans la coll. «Folio ») qui vient confirmer le rapport d'interlocution de texte à texte. Le roman de Gide est en effet connu pour contenir une mise en abyme archétypale bien qu'aporétique, puisqu'y est représentée une figure d'écrivain, Edouard, travaillant à un roman intitulé Les Faux-monnayeurs, à propos duquel il se répand en considérations techniques consignées dans son journal, en fait dans la matérialité de plusieurs carnets. Edouard, le prénom de Monsieur Songe, dont l'œuvre, autoréférentielle s'il en est, consiste en un journal, consigné dans ses (trois) carnets...

12 En effet, au moins pour nombre de lecteurs français, la désignation du personnage, monsieur Songe, évoque sans doute le Monsieur Teste (Paris, Gallimard, 1946, réédité dans la coll. «L'Imaginaire ») de Paul Valéry - avec qui Pinget a de commun la volonté de dénoncer toutes superstitions littéraires. 
présence d'une quelconque polyphonie: cette fréquente dérive métaphorique péchant par manque de rigueur heuristique $^{13}$, mieux vaut, me semble-t-il, se borner à parler de polyvocalité - l'un des facteurs de la mise en crise de la voix narrative.

\section{Ressources linguistiques : une écriture des possibles}

Y concourent également, à des degrés variables, diverses ressources linguistiques : ainsi surtout de l'usage de l'infinitif jussif, dans la mesure où il provoque une apparente disparition du locuteur: "Parler des séances de coiffeur » (p. 37), "Faire se rencontrer au cours d'une fête de village monsieur Songe et une bande de jeunes qui l'invitent à leur table. " (p. 53), etc. Bien que l'évacuation du pronom personnel ne suffise pas à légitimer l'hypothèse d'un énoncé sans énonciateur, cette «ellipse » complique tout de même sa localisation. D'autant plus que le procédé est presque systématiquement complété dans Charrue par l'emploi du conditionnel, l'usage du système hypothétique, le recours aux tours interrogatifs et à la modalisation. Certes, la convergence de ces procédures tend principalement à frapper la narration d'éventualité dans l'espoir de préserver « le foisonnement des possibles » (p. 33), mais cette substitution permanente du virtuel à l'actuel n'en contribue pas moins elle aussi au brouillage de l'origine locutive.

\section{Allusions/ironies métatextuelles}

Le phénomène est particulièrement sensible, car amplifié, dans les nombreuses séquences métatextuelles, en particulier dans celles qui se constituent en espace spécifique: les "notes roman ». Un exemple permettra de dégager cette spécificité :

Note roman.

Pour suppléer à l'intrigue, étoffer le texte, accumulation de petits faits vécus, le tout accompagné de commentaires

13 A ce propos, voir les objections rigoureuses formulées par Eric PRIÉTO, "Recherches pour un roman musical (L'Exemple de Passacaille, de Robert Pinget) », Paris, Poétique, n 94, avril 1993, et particulièrement la page 157. 
sur les aléas de l'existence, ou même pimenté d'interprétations psychologiques. Important la psychologie. Et glisser ça et là un terme scientifique, une référence littéraire, une interférence du narrateur, bref de quoi alimenter la réflexion d'un lecteur qui se veut averti. Une certaine obscurité, fruit d'un raisonnement mal conduit ou d'une syntaxe alambiquée, ne nuira pas à l'affaire. Et puis se rattraper par une phrase délicieuse qui ouvre sur des perspectives lointaines, évanescentes autant que nostalgiques. (p. 59-60)

S'il s'agit clairement de métatextuel puisque le texte assure "dans son corps même la désignation de tout ou partie de ses mécanismes constitutifs ${ }^{14}$, il serait peu pertinent d'identifier ici une mise en abyme, en raison notamment de la dimension fragmentaire de Charrue qui contrarie ce phénomène d'enchâssement de type analogique. Pour en rendre compte avec quelque rigueur, sans doute est-il nécessaire de recourir à la rhétorique métatextuelle dont Bernard Magné a jeté les bases (op. cit.), ce qui permet de caractériser cette séquence comme une allusion métatextuelle. Conformément à la définition de l'allusion par Fontanier ${ }^{15}$, dans notre passage, le locuteur " effacé » fait sentir sans le dire le rapport de la pratique d'écriture qu'il présente comme encore virtuelle avec celle déjà actualisée par Charrue - entre autres ouvrages de Pinget. Et puisque le niveau de dénotation concerne déjà du texte, le mécanisme connotatif de l'allusion doit être considéré comme métatextuel. A moins qu'on identifie ici une ironie métatextuelle, la détection de l'analogie entre écriture virtuelle et actuelle reposant nécessairement sur une prise de conscience des particularités scripturales de Charrue, qui fournissent le contexte vicaire ${ }^{16}$ indispensable à

14 Bernard MAGNÉ, "Métatextuel et lisibilité », Protée, Québec, vol. 14, n 1-2, printemps-été 1986, p. 77.

15 Pierre FONTANIER, Les Figures $d u$ discours (1830), Paris, Flammarion, 1977, coll. "Champs " (édition introduite par Gérard Genette), p. 125 : "L'Allusion [...] consiste à faire sentir le rapport d'une chose qu'on dit avec une autre qu'on ne dit pas, et dont ce rapport même réveille l'idée." 
la production de l'effet d'ironie. Mais ces deux figures ne me paraissent pas inconciliables, qui au contraire fonctionnent ici simultanément.

\section{Démultiplication locutive}

Ces phénomènes ne peuvent être dissociés du problème de la voix narrative car, on vient de le voir, c'est en partie l'indécision affectant l'origine de la parole qui les génère. De même, lorsqu'au détour d'une nouvelle expression régionale " D'une situation pénible dont on ne sait comment se sortir, [il dira] c'est la charrue à chien » (p. 30) - certains lecteurs reconnaissent une auto-impli-citation du titre de l'ouvrage où figure la formule, ils devraient être conduits à s'interroger sur le statut de l'énonciateur. C'est que dans Charrue l'instance en charge de la parole (j'ose à peine dire « narrative ») fait l'objet d'une vertigineuse démultiplication, dont l'origine réside dans l'application de la troisième personne du singulier à une expression de soi. Pinget provoque ainsi la collision de deux formes canoniques, et en imposant le spectacle de leur paradoxal métissage propose aux lecteurs de s'ouvrir à la possibilité d'une œuvre neuve, transgénérique : quelque chose comme un autoportrait inavoué (désavoué), morcelé et hybride, à la troisième personne du singulier.

A cela s'ajoutent les phénomènes de démultiplication déjà mentionnés : informés depuis Le Harnais des choix pronominaux de monsieur Songe qui «continue à se demander si parler de lui à la troisième personne est judicieux » (p. 14), il nous faut d'emblée distinguer entre « il $\mathrm{n}^{\circ} 1$-monsieur Songe-personnage » et «il $n^{\circ} 2$-monsieur Songe-narrateur »-deux instances dont nous ne saurions être fondés à gommer le dédoublement, quand bien même nous le pressentons arbitraire. De plus, monsieur Songe compose une figure d'écrivain, mais nous apprenons qu'il s'efforce de prendre « ses distances par rapport à un monsieur Songe-narrateur » (Monsieur Songe, p. 82), ce qui occasionne

16 Philippe HAMON, L'Ironie littéraire (Essai sur les formes de l'écriture oblique), Paris, Hachette, 1996, coll. « Hachette Supérieur ", p. 152 et passim. 
un nouveau dédoublement, correspondant à un degré supérieur de démultiplication : monsieur Songe-narrateur $n^{\circ} 2$ se distinguerait, ou tenterait de se distinguer, de monsieur Songe-narrateur $n^{\circ} 1$.

Et ça n'est pas tout : si l'on peut éprouver la tentation d'attribuer la paternité de Charrue à l'un quelconque des avatars schizophrènes de monsieur Songe, une différence essentielle vient tout de même faire obstacle : monsieur Songe compose une figure d'écrivain en activité, occupé à la rédaction de « ce qu'il appelle ses mémoires » (p. 31), mais ce "récit » d'une genèse non encore parvenue à son terme, nous le lisons dans un ouvrage achevé. D'où la détection d'une nouvelle instance, responsable de la structuration, de l'organisation actuelle du recueil ; dans laquelle rien n'interdit d'identifier un quatrième avatar de monsieur Songe, que sa mainmise sur l'agencement de la parole narrative inciterait à désigner comme «monsieur Songe ${ }^{\circ} 4$-scripteur » ou, dans la terminologie genettienne, monsieur Songe-auteur implicite ou plutôt induit ${ }^{17}$.

Ces distinguos « subtils » peuvent paraître fort artificiels voire arbitraires, mais ils sont en quelque sorte dictés par l'impossibilité d'investir d'un sens stable, cohérent et unitaire cette troisième personne appliquée à une expression de soi. Car c'est bien d'une quête de la personne qu'il s'agit ici, personne dont les phénomènes de démultiplication que je viens de passer en revue tendraient à ménager la pudeur. C'est par exemple le choix interprétatif qu'effectuait Marianne Alphant, délaissant la scène énonciative pour rechercher aux confins du littéraire, dans l'espace biographique, cette origine locutive unitaire qui mettrait enfin un terme à la fuite du sens sous forme d'une identité. Rendant compte de Charrue au moment de sa publication, elle intitulait sa recension critique «Monsieur Pinget qui songe »18, et soulignait maintes analogies entre la créature fictive et son créateur. L'assimilation peut certes faire bondir, mais elle ne

17 Sur ces distinctions, voir Nouveau Discours du récit, op. cit., p. 95 sq.

18 Marianne ALPHANT, « Monsieur Pinget qui songe », Libération du 5 avril 1985, p. 15. 
me semble pas entièrement illégitime, en raison du traitement systématiquement déceptif du pronom de la 3ème personne, comme de la multiplication des effets d'allusion ou/et d'ironie métatextuelles, et plus généralement des ressources connotatives de la langue qui, si nulle part n'est conclu de pacte autobiographique, tendent néanmoins en permanence à le suggérer - tout en le déjouant...

\section{L'Ennemi}

Toutefois, il n'est pas certain que cette effraction de la clôture textuelle, repérant dans l'auteur-Pinget la personne unitaire qui orchestrerait la démultiplication et le brouillage des voix, suffise à mettre un terme à la fuite du sens. En effet, depuis la divulgation et la vulgarisation des concepts de la psychanalyse est apparue une théorie du sujet différente de la morale humaniste classique, et loin d'être unitaire, ce sujet nous apparaît aujourd'hui à plus d'un titre comme clivé. Et force est de constater que les clivages en question viennent encore compliquer la scène énonciative de Charrue, où ils se répercutent :

Note roman.

Quelque chose ou quelqu'un d'innommé se rebiffe. Qui ne cadre pas avec cette sorte d'écrit. C'est très vague mais déjà troublant. Comment s'en défendre ? Ne pas se barricader, ouvrir les portes. Que se manifeste l'inconnu. Monsieur Songe devra s'effacer c'est probable. Ne sera plus qu'observateur d'un phénomène qui le dépasse. Qui en fera la relation? Et que deviendront ces notes ? (p. 78-79)

Il est certes possible de proposer une analyse strictement immanente du phénomène : il s'agirait, conformément à l'hypothèse proposée par la grammaire transformationnelle, d'une créativité ou productivité qui change les règles, à retrouver dans le texte en l'absence de recherche par l'écrivain d'un effet - voire en opposition à telle recherche. Ce naufrage du texte et plus précisément de sa dimension énon- 
ciative dans l'incertitude relèverait de ce que Julia Kristeva a analysé comme "productivité-dite-texte »19.

Mais la fréquentation assidue de l'œuvre pingétienne incite, qu'on s'en réjouisse ou s'en afflige, à déborder cette approche exclusivement technico-formaliste : Jean-Claude Vareille ${ }^{20}$, par exemple, met ainsi en évidence l'importance de la parousie dans cette œuvre, ainsi que de divers procédés alchimiques ( obscurum per obscurius »), et dans cette réexploitation d'archétypes identifie un projet d'ordre eschatologique qui relèverait donc d'une forme de mysticisme - à tout le moins de transcendance. Confirmée ${ }^{21}$ par la consultation des (rares) épitextes théoriciens autographes de Pinget ${ }^{22}$, où il confie par exemple sa fascination pour l'Augustinisme, comme pour les travaux de Jung.

\section{Quelle(s) voie(s) pour l'analyse de la voix ?}

Mon propos n'est nullement de récuser la pertinence d'une lecture immanente de l'œuvre pingétienne, privée du recours à un épitexte autographe trop souvent réservé à un cercle de " happy few ». Mais après avoir pris connaissance d'un tel épitexte, et quoi qu'on pense du « tabou de compétence sur l'interprétation auctoriale ${ }^{23}$, il devient difficile de ne pas porter sur l'œuvre un regard modifié. Et ce qui est au cœur de cette modification de la posture lectorale, c'est bien le problème de l'intention d'auteur, restauré ici dans toute sa complexité : si j'ai choisi l'exemple de Charrue, c'est que

19 Julia KRISTEVA, Semeiotikè (Recherches pour une sémanalyse), Paris, Seuil, 1969, réédition dans la coll. « Points».

20 Jean-Claude VAREILLE, «Robert Pinget - Pléthore et ascèse ", Paris, Le Nouveau Roman en questions, $\mathrm{n}^{\circ} 2$, 1993, p. 19-44.

21 Et seulement confirmée, car il est tout à fait possible d'aboutir à de telles conclusions sans consultation de l'épitexte auctorial.

22 Robert Pinget à la lettre / Entretiens avec Madeleine Renouard, Paris, Belfond, 1993. Voir également, Pinget (Robert), «Pseudoprincipes d'esthétique ", dans Nouveau Roman : hier, aujourd'hui, II : pratiques, colloque de Cerisy-la-Salle, Paris, U.G.E., 1972, coll. «10/18».

23 Gérard GENETTE, Seuils, Paris, Seuil, 1987, coll. « Poétique », p. 337 . 
Pinget, proche en cela des autres « nouveaux romanciers », a fréquemment affirmé ne pas écrire pour délivrer un message dont il aurait une conscience préalable, mais tout au contraire pour découvrir durant l'activité d'écriture ce qu'il avait à dire/écrire. Mais cela n'invalide pas l'existence d'une intention, sans conscience certes, et que l'on pourrait définir comme une forme d'intentionnalité diffuse ${ }^{24}$.

Et c'est précisément ce dont les outils d'analyse de la voix narrative proposés par Genette et reconduits par ses successeurs ne permettent pas de rendre compte. Fort d'un recul critique d'une vingtaine d'années, je confesse avoir aujourd'hui du mal à comprendre l'exclusion rigoureuse de l'auteur des frontières du texte, non pas en tant que personne physique émargeant sur les listes de l'Etat civil (ce qui va de soi) mais en tant que support d'intentions et d'affects; et les jongleries que nécessite la maîtrise des concepts d'auteur impliqué, implicite et induit me paraissent l'indice des limites de leur pertinence opératoire. Je n'oublie pas le contexte d'émergence de cette exclusion, fortement influencé par les prises de position antérieures d'un Roland Barthes qui, à une époque plus polémique encore (1968), avait été conduit à proclamer «la mort de l'auteur » 25 . Mais à présent, il me semble important dans un souci scientifique de ménager de nouveau à l'auteur quelque place dans l'élaboration des sens qui circulent dans le texte comme à sa périphérie : car cette zone frontalière est cruciale pour la compréhension de nombre d'œuvres récentes ${ }^{26}$.

24 Antoine COMPAGnon, Le Démon de la théorie (Littérature et sens commun), Paris, Seuil, 1998, coll. "La couleur des idées", chapitre 2, «L'Auteur », p. 49-99.

25 Roland BARTHES, "La Mort de l'auteur » (1968), repris dans Le Bruissement de la langue, Essais critiques IV, Paris, Seuil, 1984, réédité dans la coll. « Points ».

26 Par exemple les Romanesques de Robbe-Grillet (Le Miroir qui revient, Paris, Minuit, 1984 / Angélique ou l'enchantement, Paris, Minuit, 1988 / Les Derniers jours de Corinthe, Paris, Minuit, 1994), le dernier texte de Jean-Philippe Toussaint (Autoportrait (A l'étranger), Paris, Minuit, 2000), ou encore les plus récentes fictions d'Antoine Volodine (Vue sur l'ossuaire, Paris, Gallimard, 1998 / Le Post-exotisme en dix leçons, leçon onze, Paris, Gallimard, 1998 / Des Anges mineurs, 
Et elle engage bien sûr le symétrique de l'auteur, le ou plutôt les récepteurs du texte - car l'hypothèse d'un lecteur abstrait et idéel ne me semble qu'à demi convaincante. Le cadre nécessairement restreint de mon propos ne m'a pas permis d'analyser ces phénomènes, mais dans Charrue le statut du destinataire est également et symétriquement problématique : il faudrait ainsi distinguer entre " narratairepersonnage » (Mortin, destinataire explicite et privilégié), " narrataire invoqué » (le « lecteur qui se veut averti ») et "narrataire effacé »; et je ne suis pas certain que les notions de lecteur virtuel, implicite, abstrait ou encore Modèle suffiraient (plus généralement suffisent) à rendre compte rigoureusement des mécanismes à l'œuvre dans le cadre d'une réception concrète. Pour ce faire, sans doute est-il nécessaire de déborder la stricte scène énonciative, et de forger de nouveaux outils d'analyse, complémentaires de ceux que propose la poétique - comme ont pu le faire Michel Picard ${ }^{27}$ ou Vincent Jouve 28 .

L'analyse de la frontière entre texte et hors-texte, entre monde que l'on représente et monde où l'on représente et reçoit ces représentations, ne pourra se faire sans excéder le cadre strict de la poétique, mais l'heure n'est plus (ou moins) aux querelles de chapelles, et à tout prendre il vaut sans doute la peine de courir le risque de l'excommunication. En prenant garde toutefois de ne pas régresser, à l'inverse, du "textualisme le plus ténu [au] biographisme le plus convenu ${ }^{29}$ : l'effraction de la clôture textuelle ne doit pas devenir une brèche où se réengouffreraient les amateurs de

Paris, Seuil, 1999, coll. «Fiction \& Cie»)-liste évidemment non limitative...

27 Michel PICARD, La Lecture comme jeu, Paris, Minuit, 1986 ; et Lire le temps, Paris, Minuit, 1989 - où il pose les notions de «liseur ", « lectant » et « lu».

28 Vincent JOUVE, L'Effet-personnage dans le roman, Paris, P.U.F., 1992 ; et La Lecture, Paris, Hachette, 1993, coll. "Contours littéraires " - où il complète la typologie de Picard en introduisant la notion de « lisant ».

29 Benoît PEETERS, «Echafaudages ", Actes du colloque de Cerisy du mois de juillet 1984, repris dans Cahiers Georges Perec I, Paris, P.O.L., 1985, p. 185. 
traque aux biographèmes et autres idolâtres artistiques en tous genres. En amont, la pragmatique et l'herméneutique, en aval, les diverses théories de la réception ont déjà proposé des options alternatives entre ces écueils antagonistes : c'est sans doute à ces conditions, en retenant les leçons de ces tentatives sans pour autant répudier l'intégralité des outils forgés par les poéticiens, que chacun d'entre nous pourra trouver la voie. 